HETEROCYCLES, Vol. 71, No. 9, 2007, pp. 2049 - 2054. @ The Japan Institute of Heterocyclic Chemistry

Received, 27th April, 2007, Accepted, 11th June, 2007, Published online, 12th June, 2007. COM-07-11092

\title{
SYNTHESIS OF NEW HETEROCYCLIC SYSTEM:
}

\section{1(5)H-1,5-DIAZACYCL[3.3.2]AZINE-2,4-DIONE}

\section{Anton V. Dolzhenko, Anna V. Dolzhenko, and Wai-Keung Chui*}

Department of Pharmacy, Faculty of Science, National University of Singapore, 18 Science Drive 4, Singapore 117543, Singapore E-mail: phacwk@nus.edu.sg

Abstract - A synthesis of hitherto unknown heterocyclic system 1(5)H-1,5-diazacycl[3.3.2]azine-2,4-dione (5) is presented. The cyclocondensation of 2,6-diaminopyridine (1) with maleic anhydride yielded (5-amino-2-oxo-2,3-dihydroimidazo[1,2-a]pyridin-3-yl)acetic acid (2), which was converted to methyl (5-amino-2-oxo-2,3-dihydroimidazo[1,2-a]pyridin-3-yl) acetate hydrochloride (3). The ester (3) upon treatment with sodium carbonate solution underwent intramolecular cyclization to 2a,3-dihydro-1(5)H-1,5-diazacycl[3.3.2]azine-2,4-dione (4), which was oxidized in alkaline condition to provide 5 .

\section{INTRODUCTION}

Cyclazines are common subjects in theoretical and experimental studies. ${ }^{1}$ The diazacycl[3.3.2]azines, which contain the imidazo[1,5- $a$ ]pyridine pharmacore, can be categorized into three types of systems (I-III) (Figure). To date only one report ${ }^{2}$ on the synthesis of 1,3-diazacycl[3.3.2]azines (I) has appeared. The chemistry of 1,4-diazacycl[3.3.2] azines (II) has been further developed, ${ }^{3}$ but there is no information on the 1,5-diazacycl[3.3.2] azine heterocyclic system (III) available.

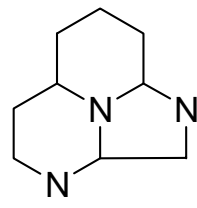

I<smiles>C1CC2CNCC3CNCC(C1)N23</smiles>

II<smiles>C1CC2CCCC3NCC(C1)N23</smiles>

III

Figure

As a continuation to our program on the reactions of maleic anhydride with binucleophiles, ${ }^{4}$ particularly in the synthesis of new heterocyclic systems, ${ }^{5}$ we describe herein a method for the preparation of 
1,5-diazacycl[3.3.2]azine system (III) derivative, namely 1(5)H-1,5-diazacycl[3.3.2]azine-2,4-dione (1,5,8b-triazaacenaphthylen-2,4-dione).

\section{RESULTS AND DISCUSSION}

The reaction of 2-aminopyridines with maleic anhydride has been shown to afford (2-oxo-2,3-dihydroimidazo[1,2-a]pyridin-3-yl)acetic acid. ${ }^{6}$ Using a modification of this method we prepared amino acid (2) from 2,6-diaminopyridine (1) and maleic anhydride (Scheme 1). The tautomeric form 2A solely was observed in the NMR spectra (DMSO- $d_{6}$ solution). However, H-3 signal in ${ }^{1} \mathrm{H}$ NMR spectrum disappeared upon addition of $\mathrm{D}_{2} \mathrm{O}$ to DMSO- $d_{6}$ solution of 2 that indicated keto-enol tautomerism in the compound (2). The ${ }^{1} \mathrm{H}$ NMR spectrum of 2 in $\mathrm{D}_{2} \mathrm{O}$ contained no $\mathrm{H}-3$ signal, but the signals of the methylenic group appeared as AB system (instead of AMX system in DMSO- $d_{6}$ ). This type of splitting confirmed diastereotopicity of the methylenic group protons that corresponded to the form $\mathbf{2 A}$. The signal of C-3 in ${ }^{13} \mathrm{C}$ NMR spectrum appeared as an equally populated triplet at $62.6 \mathrm{ppm}\left({ }^{2} J_{\mathrm{CD}}=22.9 \mathrm{~Hz}\right)$, which was observed in $\mathrm{D}_{2} \mathrm{O}$ solution, also supported the existence of the form $2 \mathrm{~A}$.

Attempts of the direct thermal intramolecular cyclization of 2 were not successful. Therefore, the acid (2) was treated with thionyl chloride in methanol to afford 3. The tautomerism, similar to the compound (2), was observed for the ester (3). Interestingly, N-1 atom was found to be protonated in the compound (3) as indicated by cross-peaks between NH (12.89 ppm) and H-8 (6.40 ppm) signals in 2D NOESY experiment.

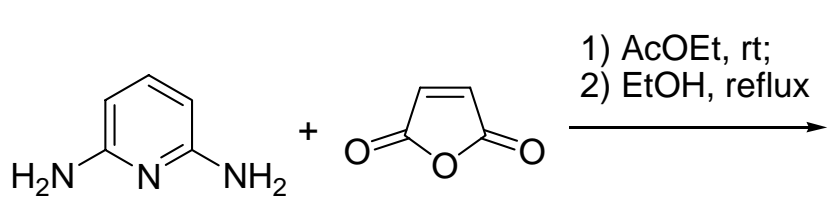

1

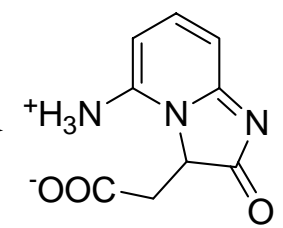

$2 A$

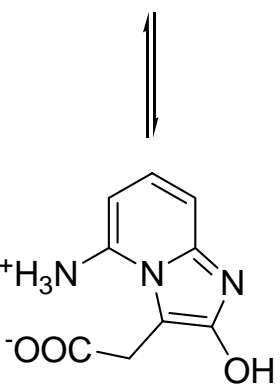

2B

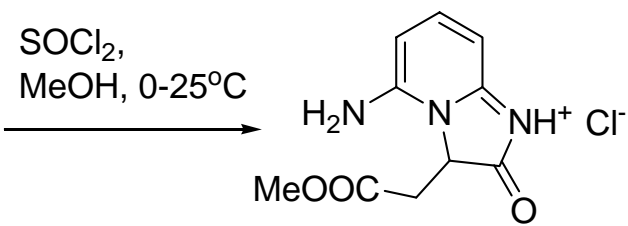

3A

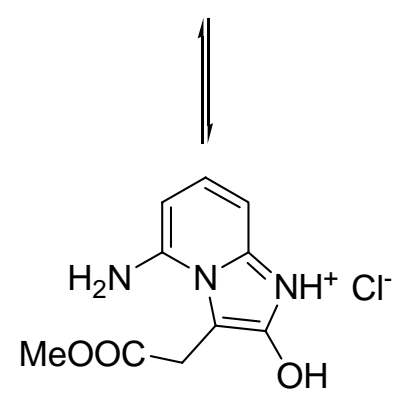

3B

Scheme 1

The ester (3) upon treatment with sodium carbonate solution in mild condition underwent intramolecular ring closure and provided the tricyclic system 2a,3-dihydro-1(5)H-1,5-diazacycl[3.3.2]azine-2,4-dione (4) (Scheme 2). The compound (4) was readily enolized in alkaline condition and oxidized in aqueous solution by standing exposed to air overnight. After treatment of the resulting product with acetic acid, 1(5)H-1,5-diazacycl[3.3.2]azine-2,4-dione (5) was isolated and characterized. Annular tautomerism (forms 
5A and 5B) was found to be possible along with keto-enol tautomerism. However, we were not able to distinguish between these forms using the spectral data and X-ray crystallographic study might be required.

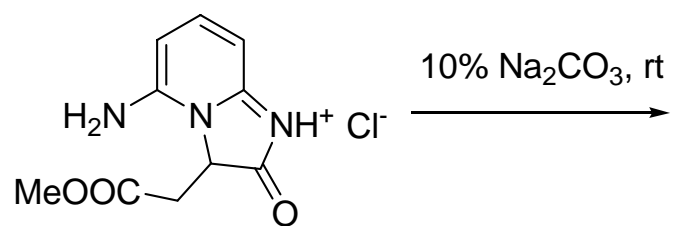

3<smiles>O=C1CC2C(=O)N=C3C=CC=C(N1)N32</smiles>

$4 \mathrm{~A}$
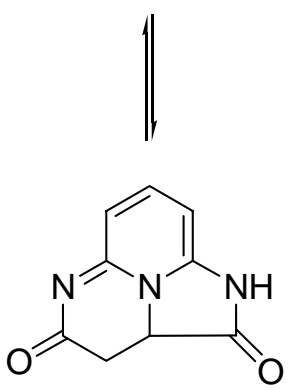

4B
1) $[\mathrm{O}]$ (air), $3 \% \mathrm{Na}_{2} \mathrm{CO}_{3}, \mathrm{rt}$;

2) $\mathrm{AcOH}, \mathrm{rt}$<smiles>O=c1cc2c(=O)nc3cccc([nH]1)-n-3-2</smiles>

$5 \mathrm{~A}$

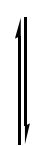<smiles>O=c1cc2c(=O)[nH]c3cccc(n1)n32</smiles>

5B

Scheme 2

In conclusion, an efficient and simple synthesis of new cyclazine type heterocyclic system viz. 1(5)H-1,5-diazacycl[3.3.2]azine-2,4-dione was successfully developed. Further investigation of the 1(5)H-1,5-diazacycl[3.3.2] azine chemistry is in the progress.

\section{EXPERIMENTAL}

General Methods. Melting points (uncorrected) were determined on a Gallenkamp melting point apparatus. IR spectra were performed on a Shimadzu IRPrestige- 21 spectrophotometer in $\mathrm{KBr}$ discs. ${ }^{1} \mathrm{H}$ and ${ }^{13} \mathrm{C}$ NMR spectra were recorded on a Bruker DPX-300 spectrometer, using DMSO- $d_{6}$ or $\mathrm{D}_{2} \mathrm{O}$ as solvents and TMS or TSP as internal references. The assignments were made using the reported data for (2-oxo-2,3-dihydroimidazo[1,2-a]pyridin-3-yl)acetic acids, ${ }^{6}$ DEPT135, 2D COSY, NOESY and HMQC spectral data.

\section{(5-Amino-2-oxo-2,3-dihydroimidazo[1,2-a]pyridin-3-yl)acetic acid (2).}

The freshly recrystallized from toluene 2,6-diaminopyridine (1, 2.18 g, $20 \mathrm{mmol})$ was dissolved in EtOAc $(40 \mathrm{~mL})$ and a solution of maleic anhydride $(1.96 \mathrm{~g}, 20 \mathrm{mmol})$ in EtOAc $(20 \mathrm{~mL})$ was slowly added with stirring at $0^{\circ} \mathrm{C}$ (ice bath). After stirring at $0^{\circ} \mathrm{C}$ for $30 \mathrm{~min}$, ice bath was removed and stirring was continued for $12 \mathrm{~h}$ at $\mathrm{rt}$. The precipitate was filtered, washed with EtOAc and refluxed in EtOH for 30 min. After cooling, the product (2) was filtered, washed with EtOH and recrystallized from water to give sandy crystals. Yield $2.82 \mathrm{~g}(68 \%) ; \mathrm{mp}>360^{\circ} \mathrm{C}$ (water).

IR (KBr, v, $\left.\mathrm{cm}^{-1}\right): 3550,3466,3369,3102,1727,1682,1634,1591,1551,1407$. 
${ }^{1} \mathrm{H}$ NMR (300 MHz, DMSO- $\left.d_{6} / \mathrm{TMS}\right): \delta 2.73\left(1 \mathrm{H}, \mathrm{dd},{ }^{3} J_{\mathrm{AX}}=4.5 \mathrm{~Hz},{ }^{2} J_{\mathrm{MX}}=17.7 \mathrm{~Hz}, \mathrm{H}_{\mathrm{X}}\right), 3.14(1 \mathrm{H}, \mathrm{dd}$, $\left.{ }^{3} J_{\mathrm{AM}}=3.8 \mathrm{~Hz},{ }^{2} J_{\mathrm{MX}}=17.7 \mathrm{~Hz}, \mathrm{H}_{\mathrm{M}}\right), 4.68\left(1 \mathrm{H}, \mathrm{dd},{ }^{2} J_{\mathrm{AM}}=3.8 \mathrm{~Hz},{ }^{2} J_{\mathrm{AX}}=4.5 \mathrm{~Hz}, \mathrm{H}-3\right), 6.16\left(1 \mathrm{H}, \mathrm{d},{ }^{3} J=8.3\right.$ $\mathrm{Hz}, \mathrm{H}-8), 6.19\left(1 \mathrm{H}, \mathrm{d},{ }^{3} J=8.3 \mathrm{~Hz}, \mathrm{H}-6\right), 7.54\left(1 \mathrm{H}, \mathrm{t},{ }^{3} \mathrm{~J}=8.3 \mathrm{~Hz}, \mathrm{H}-7\right), 8.30\left(3 \mathrm{H}, \mathrm{br} . \mathrm{s}, \mathrm{NH}_{3}{ }^{+}\right)$.

${ }^{13} \mathrm{C}$ NMR (75 MHz, DMSO-d $/$ / TMS): $\delta 34.4\left(\mathrm{CH}_{2}\right), 58.7$ (C-3), 94.7 (C-8), 99.4 (C-6), 143.0 (C-7), 151.5 (C-5), 158.1 (C-8a), 171.5 (C-2), $178.4\left(\mathrm{COO}^{-}\right)$.

${ }^{1} \mathrm{H}$ NMR (300 MHz, D $\left.\mathrm{O} / \mathrm{TSP}\right): \delta 3.06\left(1 \mathrm{H}, \mathrm{d},{ }^{2} J_{\mathrm{AB}}=17.7 \mathrm{~Hz}, \mathrm{H}_{\mathrm{A}}\right), 3.33\left(1 \mathrm{H}, \mathrm{d},{ }^{2} J_{\mathrm{AB}}=17.7 \mathrm{~Hz}, \mathrm{H}_{\mathrm{B}}\right), 6.48$ $\left(1 \mathrm{H}, \mathrm{d},{ }^{3} \mathrm{~J}=7.9 \mathrm{~Hz}, \mathrm{H}-8\right), 6.63\left(1 \mathrm{H}, \mathrm{d},{ }^{3} \mathrm{~J}=8.7 \mathrm{~Hz}, \mathrm{H}-6\right), 7.79\left(1 \mathrm{H}, \mathrm{t},{ }^{3} \mathrm{~J}=8.3 \mathrm{~Hz}, \mathrm{H}-7\right)$.

${ }^{13} \mathrm{C}$ NMR (75 MHz, D $\left.2 \mathrm{O} / \mathrm{TSP}\right): \delta 37.4\left(\mathrm{CH}_{2}\right), 62.6\left(\mathrm{t},{ }^{1} J_{\mathrm{CD}}=22.9 \mathrm{~Hz}, \mathrm{C}-3\right), 97.7(\mathrm{C}-8), 108.1$ (C-6), 147.5 (C-7), 152.0 (C-5), 154.7 (C-8a), 177.6 (C-2), $178.3\left(\mathrm{COO}^{-}\right)$.

Anal. Calcd for $\mathrm{C}_{9} \mathrm{H}_{9} \mathrm{~N}_{3} \mathrm{O}_{3}$ : C, 52.17; H, 4.38; N, 20.28. Found: C, 51.85; H, 4.72; N, 20.28.

\section{Methyl (5-amino-2-oxo-2,3-dihydroimidazo[1,2-a]pyridin-3-yl)acetate hydrochloride (3).}

To a suspension of acid $2(2.07 \mathrm{~g}, 10 \mathrm{mmol})$ in $\mathrm{MeOH}(30 \mathrm{~mL})$, thionyl chloride $(1.46 \mathrm{~mL}, 20 \mathrm{mmol})$ was added slowly with stirring at $0^{\circ} \mathrm{C}$ (ice bath). After stirring at $0^{\circ} \mathrm{C}$ for $30 \mathrm{~min}$, ice bath was removed and stirring was continued for $4 \mathrm{~h}$ at $\mathrm{rt}$. The solvent and excess of thionyl chloride were evaporated under reduced pressure and residue was recrystallized from $\mathrm{EtOH}$ to give sandy crystalline product (3). Yield $2.45 \mathrm{~g}(95 \%) ; \mathrm{mp}>360^{\circ} \mathrm{C}(\mathrm{EtOH})$.

IR $\left(\mathrm{KBr}, \mathrm{v}, \mathrm{cm}^{-1}\right): 3372,3075,1757,1730,1675,1646,1586,1410$.

${ }^{1} \mathrm{H}$ NMR (300 MHz, DMSO-d $/$ TMS): $\delta 3.17\left(1 \mathrm{H}, \mathrm{dd},{ }^{3} J_{\mathrm{AX}}=2.7 \mathrm{~Hz},{ }^{2} J_{\mathrm{MX}}=18.1 \mathrm{~Hz}, \mathrm{H}_{\mathrm{X}}\right), 3.52(3 \mathrm{H}, \mathrm{s}$, COOMe $), 3.79\left(1 \mathrm{H}, \mathrm{dd},{ }^{3} J_{\mathrm{AM}}=4.9 \mathrm{~Hz},{ }^{2} J_{\mathrm{MX}}=18.1 \mathrm{~Hz}, \mathrm{H}_{\mathrm{M}}\right), 5.27\left(1 \mathrm{H}, \mathrm{dd},{ }^{3} J_{\mathrm{AM}}=4.9 \mathrm{~Hz},{ }^{3} J_{\mathrm{AX}}=2.7 \mathrm{~Hz}\right.$, H-3), $6.40\left(1 \mathrm{H}, \mathrm{d},{ }^{3} J=7.9 \mathrm{~Hz}, \mathrm{H}-8\right), 6.66\left(1 \mathrm{H}, \mathrm{d},{ }^{3} J=8.7 \mathrm{~Hz}, \mathrm{H}-6\right), 7.81\left(1 \mathrm{H}, \mathrm{t},{ }^{3} J=8.1 \mathrm{~Hz}, \mathrm{H}-7\right), 8.82(2 \mathrm{H}$, s, $\left.\mathrm{NH}_{2}\right), 12.89$ (1H, br. s, NH).

${ }^{13} \mathrm{C}$ NMR (75 MHz, DMSO-d $/ 6$ TMS): $\delta 30.1\left(\mathrm{CH}_{2}\right), 52.0$ (COOMe), 57.7 (C-3), 92.7 (C-8), 104.7 (C-6), 144.3 (C-7), 148.5 (C-5), 151.7 (C-8a), 168.6 (COOMe), 170.1 (C-2).

${ }^{1} \mathrm{H}$ NMR (300 MHz, D $\left.\mathrm{O} / \mathrm{TSP}\right): \delta 3.37\left(1 \mathrm{H}, \mathrm{d},{ }^{2} J_{\mathrm{AB}}=17.7 \mathrm{~Hz}, \mathrm{H}_{\mathrm{A}}\right), 3.63(3 \mathrm{H}, \mathrm{s}, \mathrm{COOMe}), 3.68(1 \mathrm{H}, \mathrm{d}$, $\left.{ }^{2} J_{\mathrm{AB}}=17.7 \mathrm{~Hz}, \mathrm{H}_{\mathrm{B}}\right), 6.51\left(1 \mathrm{H}, \mathrm{d},{ }^{3} J=7.9 \mathrm{~Hz}, \mathrm{H}-8\right), 6.62\left(1 \mathrm{H}, \mathrm{d},{ }^{3} \mathrm{~J}=8.7 \mathrm{~Hz}, \mathrm{H}-6\right), 7.81\left(1 \mathrm{H}, \mathrm{t},{ }^{3} \mathrm{~J}=8.3 \mathrm{~Hz}\right.$, $\mathrm{H}-7)$.

${ }^{13} \mathrm{C}$ NMR (75 MHz, D $\left.2 \mathrm{O} / \mathrm{TSP}\right): \delta 33.2\left(\mathrm{CH}_{2}\right), 55.7$ (COOMe), $60.9\left(\mathrm{t},{ }^{1} J_{\mathrm{CD}}=22.6 \mathrm{~Hz}, \mathrm{C}-3\right), 98.7(\mathrm{C}-8)$, 107.8 (C-6), 148.0 (C-7), 154.2, 154.8 (C-5 and C-8a), 173.6 (COOMe), 178.2 (C-2).

Anal. Calcd for $\mathrm{C}_{10} \mathrm{H}_{12} \mathrm{~N}_{3} \mathrm{O}_{3} \mathrm{Cl}$ : C, 46.61; H, 4.69; N, 16.31. Found: C, 46.38; H, 4.87; N, 16.09.

\section{1(5)H-1,5-Diazacycl[3.3.2]azine-2,4-dione (5).}

Salt 3 (1.29 g, $5 \mathrm{mmol}$ ) was dissolved in $10 \%$ aqueous $\mathrm{Na}_{2} \mathrm{CO}_{3}(5.5 \mathrm{~mL})$ was stirred at rt. After $30 \mathrm{~min}$, the precipitated bright yellow compound 4 was filtered and washed with cold water. Without further 
purification, compound 4 was dissolved in $3 \%$ aqueous $\mathrm{Na}_{2} \mathrm{CO}_{3}(20 \mathrm{~mL})$ and stirred overnight in an open flask. To the formed suspension of sodium salt of $5, \mathrm{AcOH}(5 \mathrm{~mL})$ was added and the orange precipitate was filtered and washed with cold water. The purification of the product (5) can be performed using recrystallization from DMSO or, more conveniently, by reprecipitation from the solution in aqueous $10 \%$ aqueous $\mathrm{Na}_{2} \mathrm{CO}_{3}$ using $\mathrm{AcOH}$. Yield $0.77 \mathrm{~g}(82 \%) ; \mathrm{mp}>360^{\circ} \mathrm{C}$ (DMSO).

IR (KBr, v, $\left.\mathrm{cm}^{-1}\right): 3448,3059,1735,1699,1667,1617,1605,1485,1456,1393$.

${ }^{1} \mathrm{H}$ NMR (300 MHz, DMSO-d 6 / TMS): $\delta 6.69\left(1 \mathrm{H}, \mathrm{d},{ }^{3} J=7.9 \mathrm{~Hz}\right), 6.86\left(1 \mathrm{H}, \mathrm{d},{ }^{3} J=8.7 \mathrm{~Hz}\right), 6.99(1 \mathrm{H}, \mathrm{s})$, $7.91\left(1 \mathrm{H}, \mathrm{t},{ }^{3} J=8.3 \mathrm{~Hz}\right), 13.01(1 \mathrm{H}$, br. s. $\mathrm{NH})$.

Anal. Calcd for $\mathrm{C}_{15} \mathrm{H}_{12} \mathrm{~N}_{4} \mathrm{O}_{3}$ : C, 57.76; H, 2.69; N, 22.45. Found: C, 57.99; H, 2.82; N, 22.78 .

\section{ACKNOWLEDGEMENTS}

This work is supported by the Academic Research Fund, National University of Singapore.

\section{REFERENCES}

1. A. Taurins, 'The Chemistry of Heterocyclic Compounds: The Chemistry of Cyclazines,' Vol. 30, ed. by A. Weissberger and E. C. Taylor, John Wiley, New York, 1977, pp. 245-270; K. Matsumoto, T. Uchida, and J. Yamauchi, Yuki Gosei Kagaku Kyokaishi, 1977, 35, 739; W. Flitsch and U. Kraemer, 'Advances in Heterocyclic Chemistry: Cyclazines and Related N-Bridged Annulenes,' Vol. 22, ed. by A. R. Katritzky and A. J. Boulton, Academic Press, New York, 1978, pp. 321-365; D. Leaver, Pure Appl. Chem., 1986, 58, 143; Y. Tominaga, Y. Shiroshita, and A. Hosomi, Heterocycles, 1988, 27, 2251; Y. Matsuda, Yakugaku Zasshi, 2001, 121, 971; Y. Tominaga, 'Science of Synthesis: Product Class 7: Cyclazines,' Vol. 17, ed. by S. M. Weinreb, Georg Thieme Verlag, Stuttgart - New York, 2004, pp. 1025-1079; Y. Tominaga and K. Ueda, J. Heterocycl. Chem., 2005, 42, 337.

2. J. M. Chezal, E. Moreau, G. Delmas, A. Gueiffier, Y. Blache, G. Grassy, C. Lartigue, O. Chavignon, and J. C. Teulade, J. Org. Chem., 2001, 66, 6576.

3. K. Kurata, H. Awaya, Y. Tominaga, Y. Matsuda, and G. Kobayashi, Yakugaku Zasshi, 1978, 98, 631; M. Takatani, Y. Shibouta, K. Tomimatsu, and T. Kawamoto, WO Pat. 9602542, 1996 (Chem. Abstr., 125, 33679); M. Wakimasu and T. Ikemoto, JP Pat. 09249666, 1997 (Chem. Abstr., 127, 293222); T. Kawamoto, Y. Shibouta, M. Takatani, and M. Noda, EP Pat. 826686, 1998 (Chem. Abstr., 128, 204886); S. Imoto, M. Yoshioka, and T. Kashihara, WO Pat. 9829136, 1998 (Chem. Abstr., 129, 113515); T. Kawamoto, K. Tomimatsu, T. Ikemoto, H. Abe, K. Hamamura, and M. Takatani, Tetrahedron Lett., 2000, 41, 3447; T. Ikemoto, T. Kawamoto, K. Tomimatsu, M. Takatani, and M. Wakimasu, Tetrahedron, 2000, 56, 7915; J. S. Chun, E. Ishikawa, M. Kobayashi, and T. Kawamoto, JP Pat. 2002201193, 2002 (Chem. Abstr., 137, 93751); K. Kamiyama, N. Kanzaki, A. Hasuoka, M. Mochizuki, and T. Kawamoto, JP Pat. 2002371042, 2002 (Chem. Abstr., 138, 
55742).

4. A. V. Dolzhenko, W. K. Chui, and A. V. Dolzhenko, Heterocycles, 2006, 68, 821.

5. A. V. Dolzhenko and W. K. Chui, Heterocycles, 2004, 63, 2623.

6. A. V. Dolzhenko, N. V. Kolotova, V. O. Kozminykh, W. K. Chui, P. W. S. Heng, and V. N. Khrustalev, Heterocycles, 2004, 63, 55; M. E. Baumann, H. Bosshard, W. Breitenstein, G. Rihs, and T. Winkler, Helv. Chim. Acta, 1984, 67, 1897. 